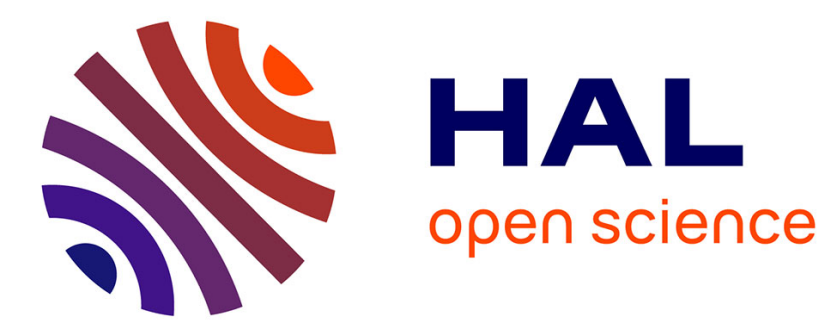

\title{
Raman evidence for the successful synthesis of diamane
}

\author{
Fabrice Piazza, Kelvin Cruz, Marc Monthioux, Pascal Puech, I.C. Gerber
}

\section{To cite this version:}

Fabrice Piazza, Kelvin Cruz, Marc Monthioux, Pascal Puech, I.C. Gerber. Raman evidence for the successful synthesis of diamane. Carbon, 2020, 169, pp.129-133. 10.1016/j.carbon.2020.07.068 . hal03091133

\section{HAL Id: hal-03091133 \\ https://hal.science/hal-03091133}

Submitted on 30 Dec 2020

HAL is a multi-disciplinary open access archive for the deposit and dissemination of scientific research documents, whether they are published or not. The documents may come from teaching and research institutions in France or abroad, or from public or private research centers.
L'archive ouverte pluridisciplinaire HAL, est destinée au dépôt et à la diffusion de documents scientifiques de niveau recherche, publiés ou non, émanant des établissements d'enseignement et de recherche français ou étrangers, des laboratoires publics ou privés. 


\section{Raman evidence for the successful synthesis of diamane}

\section{Fabrice Piazza*, K. Cruz}

Nanoscience Research Laboratory, Pontificia Universidad Católica Madre y Maestra, Autopista Duarte km 1 1⁄2, Apartado Postal 822, Santiago de los Caballeros, Dominican Republic

\section{Marc Monthioux, Pascal Puech}

Centre d'Elaboration des Matériaux et d'Etudes Structurales (CEMES), CNRS, Université de Toulouse, 29, Rue Jeanne Marvig, BP 94347, 31055, Toulouse, France

\section{Iann Gerber}

Laboratoire de Physico-Chimie des Nano-Objets (LPCNO), CNRS, INSA, Université de Toulouse, 135 Avenue de Rangueil, 31400, Toulouse, France

Diamane was prepared from the exposure of bi-layer graphene to $H$ radicals produced by the hot-filament process. A sharp $s p^{3}$-bonded carbon stretching mode was observed by UV Raman spectroscopy while no $s p^{2}$-bonded carbon peak was simultaneously detected. This is the first time that Raman spectra of genuine diamane are reported, which, meanwhile, are the very first evidence for the successful synthesis of genuine diamane. First principle calculations support possible full hydrogenation and confirm the hydrogenated AB configuration to be the most stable one. We believe those results constitute a milestone in the path towards the synthesis of high-quality diamane and open the door to large-scale production.

Genuine diamane consists of two crystalline $\mathrm{sp}^{3}$-bonded carbon layers for which half of the carbon atoms are hydrogenated while the other half bond the two layers to each other $[1,2]$. The material stability was first predicted by Chernozatonskii et al in 2009 [1]. The increasing interest in this new 2D wide bang-gap semiconducting material comes, in particular, from its potential use in electronics [3]. Recently, the synthesis of stable 2D nanometer-thick and crystalline $s p^{3}$-bonded carbon, was unambiguously shown for the first time [2,4]. This breakthrough was achieved from the chemisorption of $\mathrm{H}$ radicals produced by the hot-filament process at low temperature and pressure on the basal planes of few-layer graphene (FLG) and the subsequent interlayer bonding between $\mathrm{sp}^{3}$-bonded carbon atoms $\left(\mathrm{sp}^{3}-\mathrm{C}\right) . s p^{3}-\mathrm{C}$ stretching peak was detected in visible and UV 
Raman spectra. However, these reports cannot claim having evidenced genuine diamane, because of the poor suitably of the starting graphene material with too many layers, thereby inducing the formation of diamanoïd/graphene hybrids instead, and because of the lack of lateral resolution of Raman spectroscopy [4]. Following that work, the synthesis of F-diamane, where carbon atoms are fluorinated rather than hydrogenated as in genuine diamane, was claimed from the fluorination, at low pressure, of AB-stacked bi-layer graphene [5], the latter having been shown to be the most favorable configuration for the $\mathrm{sp}^{2}-\mathrm{C}$ to $\mathrm{sp}^{3}-\mathrm{C}$ conversion [1,2]. However, notably, no $\mathrm{sp}^{3}-\mathrm{C}$ related peak was observed in Raman spectra [5]. Also, the method based on the intensity of the electron diffraction spots to discriminate between the graphite and the diamond structures is known to be unreliable because of the "interference error" effect which can occur in the case of ultrathin films that can be easily distorted [4,6]. Hence, to the best of authors' knowledge, evidences of diamane synthesis are missing.

Raman spectroscopy is a critical technique to characterize carbon materials, including hydrogenated graphene, graphane [7] and diamanoïds [2,4]. However, in the case of those nanomaterials, since for visible energies, the Raman scattering of $s p^{2}-\mathrm{C}$ is a resonant process between 50 and 230 times more effective than that of $s p^{3}-\mathrm{C}$ [8], it is necessary to employ UV excitation to obtain a more evenly weighted probe of $s p^{3}-\mathrm{C}$ and $s p^{2}-\mathrm{C}$ sites. This critical point has not been considered in most reports on hydrogenated graphene and 2D $s p^{3}-\mathrm{C}$ nanomaterials [2].

Therefore, in this work, we studied the hydrogenation of bi-layer graphene by the chemisorption of $\mathrm{H}$ generated from the dissociation of $\mathrm{H}_{2}$ in a hot filament reactor at low temperature and low pressure, and the subsequent structure conversion into highly hydrogenated genuine diamane. UV Raman spectroscopy was used to track the structure conversion, which is unprecedented in the literature in the field but in our own work [2,4]. First principle calculations and electronic diffraction are used to explain our findings. In addition, ultra-low voltage (5 kV) transmission electron microscopy (TEM) analysis was carried-out, mostly in electron diffraction mode.

As-received suspended bi-layer (2LG) graphene films grown by chemical vapor deposition and deposited on $3 \mathrm{~mm}$ diameter gold Quantifoil TEM grids were obtained from Graphenea and used as starting graphene materials. Each film is polycrystalline, with a maximum grain size of 20 $\mu \mathrm{m}$. Coverage of the grid is $\sim 98 \%$. 2LG films were obtained from the successive transfer of two individual single-layer graphene (1LG), hence the resulting 2LG stacking is random. However, 
considering the dimension of the 1LG layers ( $\sim 3 \mathrm{~mm}$ in diameter) and their polycrystalline nature (20- $\mu \mathrm{m}$-large grains for the most), chances for locally getting the favorable AB or AA stacking configurations [1,2] at micrometer scale are high and can be actually observed as reported in Fig.1b. A commercial hot filament reactor (BWI 1000 model from Blue Wave Semiconductors) was used for the hydrogenation process [9]. The specific experimental details related to the synthesis of diamanoïds were previously disclosed [2]. 2LG grids were placed vertically on the substrate holder. The samples studied here were prepared at a pressure of 50 Torr and using a $\mathrm{H}_{2}$ flux of $1 \mathrm{sccm}$. The duration was of 6 minutes and 30 seconds. Filament temperature was within the range of $2350-2590{ }^{\circ} \mathrm{C}$ while the maximum $2 \mathrm{LG}$ temperature was around $400{ }^{\circ} \mathrm{C}$.

The Raman scattering measurements were performed in the backscattering configuration using $244 \mathrm{~nm}$ line and analyzed using a $\times 40$ objective micro-Raman spectrometer system (inVia Reflex, Renishaw). The resolution was of $3.5 \mathrm{~cm}^{-1}$. Typically, laser power was in the range of 1 $\mathrm{mW}$ with 1s exposure time. No visible damage and no change of the spectral shape during measurements have been observed. Highly-oriented pyrolytic graphite (HOPG) was used for peak position calibration. Raman mapping was employed using high-speed encoded mapping stage to generate high definition 2D chemical images over typically around 140,000 $\mu \mathrm{m}^{2}$. Step size was set at $3 \mu \mathrm{m}$.

TEM (mostly electron diffraction) was employed to further examine the material structure before and after hydrogenation. The converted material, as opposed to the behavior of graphene or diamond, appeared to be electron sensitive, possibly because of the high hydrogenation degree of the surface, even for electron energy as low as $80 \mathrm{keV}$ [2]. This is consistent with the photon sensitivity observed under certain conditions [2] and the reported electron sensitivity of F-diamane [5]. This is the reason why an ultra-low-voltage $(5 \mathrm{kV})$ benchtop transmission electron microscope from America Delong was used. The instrument includes a Schottky-type field emission gun and a $2560 \times 2160$ pixel Front Illuminated Scientific CMOS $\left(6.5 \mu \mathrm{m}^{2}\right.$ pixel size $)$. Electron diffraction patterns were obtained in selected area mode from $100 \mathrm{~nm}$-large areas.

DFT calculations were performed with the VASP package $[10,11]$ with the same conditions than in our previous work [4]: Perdew-Burke-Ernzerhof (PBE) functional [12], cut-off energy of $400 \mathrm{eV}$, force convergence below $0.005 \mathrm{eV} / \AA$, vacuum height of $20 \AA$ and supercell of (7×7) with a $7 \times 7 \times 1$ grid for k-points sampling. VESTA software was used to generate optimized structure pictures [13]. 
Selected area electron diffraction of the starting 2LG films (Fig.1b-d) exhibited various features depending on the location of the area analyzed, i.e., the evidence for (i) single graphenic domains (supposedly 2LG with $\mathrm{AB}$ or $\mathrm{AA}$ stacking) (Fig.1b); (ii) two randomly stacked superimposed graphenic domains (supposedly 1 LG each) (Fig.1c) (iii) four randomly stacked graphenic domains (Fig.1d), showing than the material is not $2 \mathrm{LG}$ only all over the grid.

Micro-Raman mapping was performed before and after exposure to the hot-filament-promoted hydrogenation process, to track any structure conversion change induced by the above-mentioned process. Before hydrogenation process, the spectra of $2 \mathrm{LG}$ are characterized by a regular sharp $\mathrm{G}$ peak at around $1582 \mathrm{~cm}^{-1}$ which is due to bond stretching of all pairs of $\mathrm{sp}^{2}-\mathrm{C}$ in graphene sheets. After the hydrogenation process, drastic changes can be observed in Raman spectra of various regions of the 2LG samples. Fig.2a presents a typical spectrum of such a region, which exhibits specific features: (i) the G peak is no longer detected; (ii) the D peak which originates from defects in graphene sheets [7] is not observed either; (iii) a sharp peak (FWHM of around $10-33 \mathrm{~cm}^{-1}$ ) at around 1344-1367 $\mathrm{cm}^{-1}$ has occurred. This type of spectrum can be observed over extended regions of several $\mu \mathrm{m}$ width and in various areas probed by the laser, as illustrated in Fig. $2 \mathrm{~b}$ which displays a map of the intensity of this peak. The peak at around $1344-1367 \mathrm{~cm}^{-1}$ is not the D peak, since the G peak is not simultaneously observed; also, it is too sharp anyway and its spectral position does not correspond to the value observed in the UV spectra of nanocrystalline graphite [14]. It is supposed to be due to bond stretching between $\mathrm{sp}^{3}-\mathrm{C}$, even if the peak was predicted at near $1320 \mathrm{~cm}^{-1}$ from ab initio calculations for diamane, and found at lower wavenumber in diamanoïds (1319.5-1337 $\left.\mathrm{cm}^{-1}\right)[2,4]$ and diamond $\left(1332 \mathrm{~cm}^{-1}\right)$. Therefore, Figs $2 \mathrm{a}$ and $2 \mathrm{~b}$ show that full $\mathrm{sp}^{2}-\mathrm{C}$ to $\mathrm{sp}^{3}-\mathrm{C}$ conversion, that is to say full conversion of $2 \mathrm{LG}$ into genuine diamane, can be obtained over $20 \mu \mathrm{m}$ large region. It is assumed that the conversion takes place in regions where graphene sheets are AB- or possibly AA-stacked, which are the most energetically favorable configurations $[1,2]$. But because of the variability in the stacking sequence all over the polygranular film, many other configurations should appear which lack of symmetry makes them out of the range of calculation. Therefore, the presence of stress or strain is expected in hydrogenated yet randomly stacked layers for which $\mathrm{C}-\mathrm{C}$ interlayer bonding cannot occur, or only partially, and at the grain boundaries between graphenic and diamane domains. The local modifications induced by the variety of interlayer bonding situation are probably at the origin of the variety of up-shifted positions of the $\mathrm{sp}^{3}-\mathrm{C}$ peak (Fig.2c). Cracks which have appeared in the 
film (Fig.1e, to be compared to Fig.1a), and layers stacked with a very slight twist angle (Fig.1f) are probably related to this occurrence of stress and strain.

In Fig.2c, one can note a coarse correlation of the $\mathrm{sp}^{3}-\mathrm{C}$ peak width with its position, which ranges from 1340 to $1370 \mathrm{~cm}^{-1}$. An upshift can be associated to strain due to the presence of several phases (diamane, graphene) in equilibrium, or to an inhomogeneous layer hydrogenation, or to a distorted bonding between two hydrogenated twisted layers. The Gruneisen parameter for optical phonon is around $1 \mathrm{~cm}^{-1} / \mathrm{GPa}$ [15] leading to a conversion factor of $3 \mathrm{~cm}^{-1} / \mathrm{GPa}$ for hydrostatic pressure. For biaxial strain, a splitting between singlet and doublet is expected and not observed, which is consistent with an average hydrostatic strain, which is an acceptable yet rough hypothesis. The in-plane lattice parameter of lonsdaleite is $0.249 \mathrm{~nm}$, and $0.252 \mathrm{~nm}$ in diamond, while it is $0.246 \mathrm{~nm}$ in graphene, leading to a strain of -1 to $-2 \%$. In graphene or diamond, $-1 \%$ is associated to a hydrostatic stress of about $10 \mathrm{GPa}$. Consequently, a wavenumber shift of $30 \mathrm{~cm}^{-1}$ is possible and corresponds to the range of the shift observed.

To test this hypothesis, we have performed first principle calculations on modified diamane structures. First, starting from the genuine diamane structure (one hydrogen atom every two carbon atoms), and then subsequently moving a hydrogen or a pair of hydrogen atoms to the neighboring $\mathrm{C}$ atoms, a spontaneous relocation of the hydrogen atoms to the right sites to form diamane is observed. So, hydrogen atoms self-organize when reacting with a graphene layer. Second, by adding extra hydrogens on $\mathrm{C}$ atoms already coordinated to 3 others carbon atoms so that two firstneighbor carbon atoms both wear a hydrogen, the excess $\mathrm{H}$ atoms always desorb spontaneously to reform $\mathrm{H}_{2}$ molecules. Over-hydrogenation is thus not favorable. Finally, while testing the case of under-hydrogenation by removing $7 \mathrm{H}$ atoms from the top layer, a strong structural reorganization occurs, see Fig.3a and Fig.3b. The $19 \mathrm{C}$ atoms involved in the extended defect tend to lose their $\mathrm{sp}^{3}$ character to recover a $\mathrm{sp}^{2}$ one, implying reductions of several C-C bond-lengths, confirmed by the histogram given in Fig.3c. The phonon polarization imposed by the light polarization is in the plane, activating phonons without physical limitation. In such a case, the linewidth is not broadened and the wavenumbers of the optical modes calculated by DFT are close to the bulk value within the uncertainty range of the calculations. Thus, the lattice parameter remains the same while very local distortions can be present which could be at the origin of both linewidth broadening and frequency changes. Therefore, our calculations show that full hydrogenation for $\mathrm{AB}$ stacking is the most stable, final material, and that significant distortions can occur upon partial hydrogenation. Thus, $s p^{2}-\mathrm{C}$ to $s p^{3}-\mathrm{C}$ conversion induces huge stresses, specifically at the 
grain boundaries, either when the $2 \mathrm{LG}$ involved does not exhibit the $\mathrm{AB}$ or $\mathrm{AA}$ stacking needed for allowing the optimized hydrogenation of the layers (in case of turbostratic or commensurate stacking indeed, only partial hydrogenation may occur, locally promoted when carbon atoms happen to locally superimposed), or in case of under-hydrogenation. This is also consistent with the stress estimated in diamanoïd/graphene hybrids [4].

To summary, we have shown that the hot-filament promoted hydrogenation process can be successfully used to produce genuine diamane from bi-layer graphene. UV Raman spectroscopy reveals the $\mathrm{sp}^{3}-\mathrm{C}$ stretching mode peak in diamane showing that using a UV excitation is critical for the characterization of such $\mathrm{sp}^{3}-\mathrm{C}$ nanomaterials. It is believed that the key for producing homogenous diamane films now only relies on starting from a good quality $2 \mathrm{LG}$ material, that is to say, ideally, single-crystal $\mathrm{AB}$ flakes as large as possible. The present results open the door to mass-produced diamane by means of the hot-filament-assisted CVD process, as it is employed for the industrial production of other $\mathrm{sp}^{3}$-bonded carbon materials, such as diamond films, for about 40 years.

\section{Acknowledgements}

This research was funded by the Ministry of Higher Education, Science and Technology of the Dominican Republic (2015, 2016 and 2018 FONDOCyT programs). F. Piazza would like to greatly acknowledge PUCMM for strong administrative support. Funding by the LabEx NEXT is acknowledged for enabling mutual scientific visits and research stays. I.G. acknowledges the Calcul en Midi-Pyrénées initiative CALMIP (Project p0812) for allocations of computer time, as well as GENCI-CINES and GENCI-IDRIS for Grant No. 2020-A008096649.

\section{References}

[1] L.A. Chernozatonskii, P.B. Sorokin, A. Kvashnin, D.G. Kvashnin, Diamane: Simulation of the Structure and Properties, J. Exp. Theor. Phys. Lett. 90 (2009) 134-138.

[2] F. Piazza, K. Gough, M. Monthioux, P. Puech, I. Gerber, R. Wiens, G. Paredes, C. Ozoria, Low temperature, pressureless $\mathrm{sp}^{2}$ to $\mathrm{sp}^{3}$ transformation of ultrathin, crystalline carbon films, Carbon 145 (2019) 10-22. 
[3] L.A. Chernozatonskii, P.B. Sorokin, A.A. Kuzubov, B.P. Sorokin, A.G. Kvashnin, D.G. Kvashnin et al., Influence of Size Effect on the Electronic and Elastic Properties of Diamond Films with Nanometer Thickness, J. Phys. Chem. C 115 (2011) 132-136.

[4] F. Piazza, M. Monthioux, P. Puech, I. Gerber, Towards a better understanding of the structure of diamanoïds and diamanoïd/graphene hybrids, Carbon 156 (2020) 234-241.

[5] P.V. Bakharev, M. Huang, M. Saxena, S.W. Lee, S.H. Joo, S.O. Park et al., Chemically induced transformation of chemical vapour deposition grown bilayer graphene into fluorinated single-layer diamond, Nature Nanotechnol. 15 (2020) 59-66.

[6] D.B. Williams, C.B. Carter, Diffraction from Small Volumes (Ch.17), in Transmission Electron Microscopy A Textbook for Materials Science, Second Edition, Springer, Boston, MA, 2009, pp.273-274.

[7] D.C. Elias, R.R. Nair, T.M.G. Mohiuddin, S.V. Morozov, P. Blake, M.P. Halsall et al., Control of graphene's properties by reversible hydrogenation: evidence for graphane, Science 323 (2009) 610-613.

[8] J. Wagner, C. Wild, P. Koidl, Resonance effects in Raman scattering from polycrystalline diamond films, Appl. Phys. Lett. 59 (1991) 779-781.

[9] F. Piazza, G. Morell, J. Beltran-Huarac, G. Paredes, M. Ahmadi, M. Guinel, Carbon nanotubes coated with diamond nanocrystals and silicon carbide by hot-filament chemical vapor deposition below $200{ }^{\circ} \mathrm{C}$ substrate temperature, Carbon 75 (2014) 113-123.

[10] G. Kresse, J. Hafner, Ab initio molecular dynamics for liquid metals. Phys Rev B 1993;47:558-561.

[11] G. Kresse, J. Furthmüller, Efficient iterative schemes for $a b$ initio total-energy calculations using a plane-wave basis set. Phys. Rev. B 54 (1996) 11169-11186.

[12] J.P. Perdew, K Burke, M. Ernzerhof, Generalized gradient approximation made simple. Phys. Rev. Lett. 77 (1996) 3865-3668.

[13] K. Momma, F. Izumi, VESTA 3 for three-dimensional visualization of crystal, volumetric and morphology data, J. Appl. Cryst. 44 (2011) 1272-1276.

[14] T. Paulmier, J.M. Bell, P.M. Fredericks, Deposition of nanocrystalline-graphite films by cathodic plasma electrolysis, Thin Sol. Films 515 (2007) 2926-2934.

[15] B.R. Wu, J.A. Xu, Zone-center modes of cubic and hexagonal diamond under high pressure: A first-principles study, Phys. Rev. B 60 (1999) 2964-2967. 

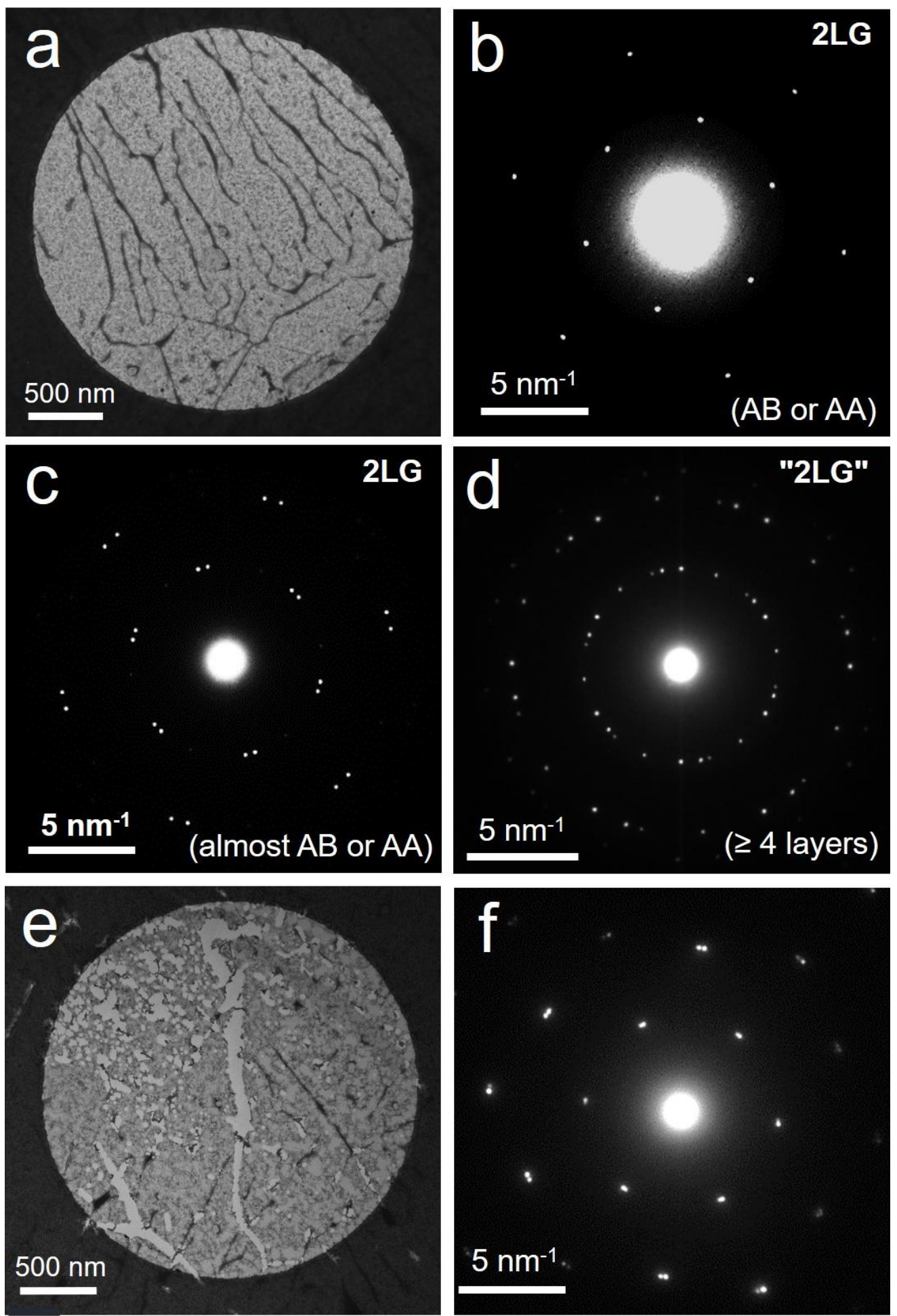

Figure 1: Examples of TEM characterization of the $2 L G$ films before (from (a) to (d)) and after hydrogenation ((e) and (f)). Diffraction patterns are from $100 \mathrm{~nm}$-large areas. (a) Image of an asreceived $2 L G$ film. Contrast variations are due to the presence of amorphous material partially covering the film. (b) Single graphenic domain, as an evidence for two superimposed $1 L G$ in AB or AA stacking; the inner and outer 6 spots correspond to the 100 and 110 spot families in graphite, respectively. (c) Two slightly rotated graphenic domains, as an example of a random stacking of two superimposed $1 L G$. (d) Evidence for the presence of 4 superimposed graphenic 
domains. (e) Image of a hydrogenated $2 L G$ film. (f) A single domain, as an evidence for the successful conversion of an $A B$ (or $A A$ )-stacked $2 L G$ domain as in (b) into a genuine diamane domain. Therefore, the inner and outer 6 spots correspond to the 111 and 110 spot families in diamond (or 100 and 110 in lonsdaleite), respectively. 

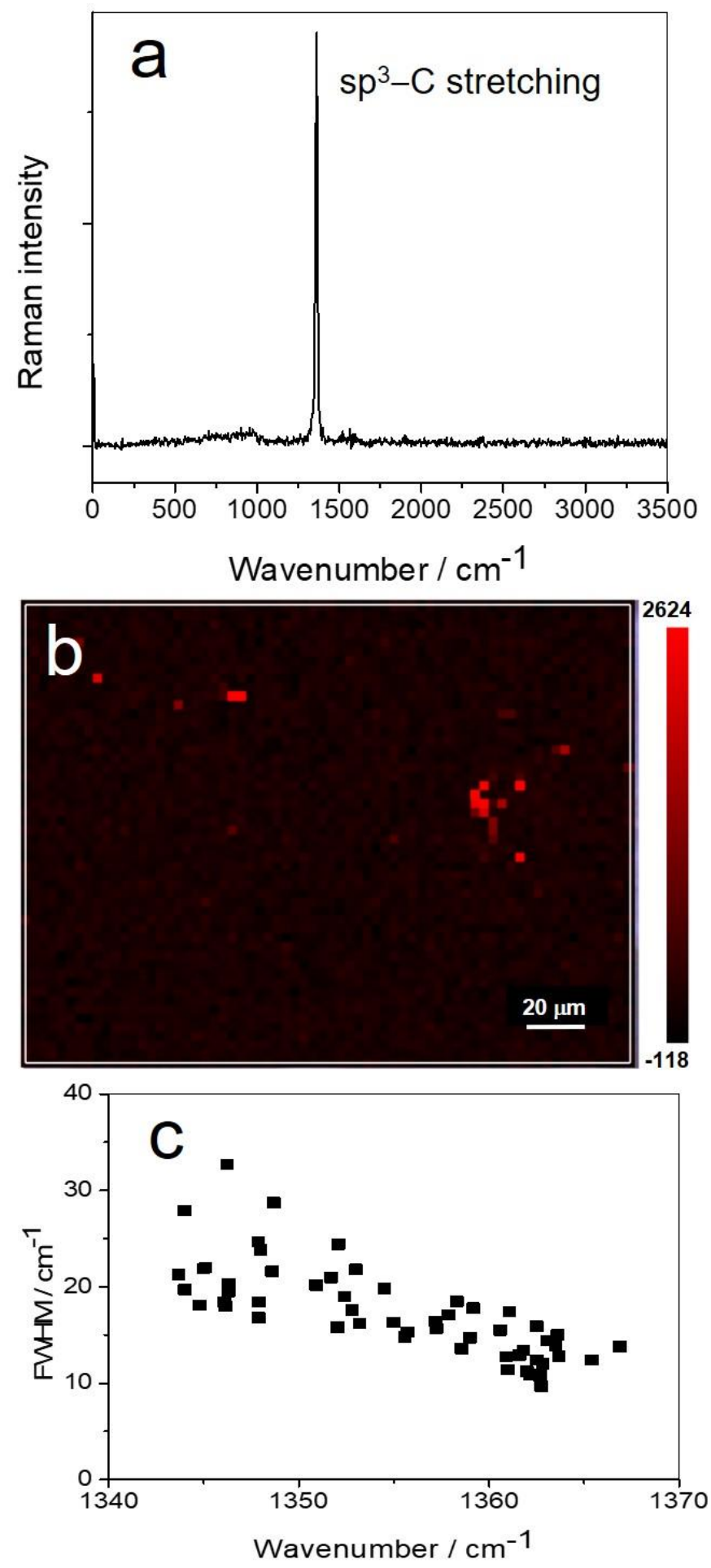

Figure 2: Typical UV (at $244 \mathrm{~nm}$ ) Raman data. (a) Example of a Raman spectrum obtained on a hydrogenated bilayer graphene, as the evidence for the occurrence of genuine diamane, as discussed in the text. (b) Example of the Raman map of the intensity of the sp ${ }^{3}-C$ stretching mode of $2 L G$ after exposure to the hot-filament-promoted hydrogenation process. (c) Full Width at Half Maximum (FWHM) as a function of wavenumber of the $\mathrm{sp}^{3}-C$ stretching peak for graphene materials exposed to the hot-filament-promoted hydrogenation process. 

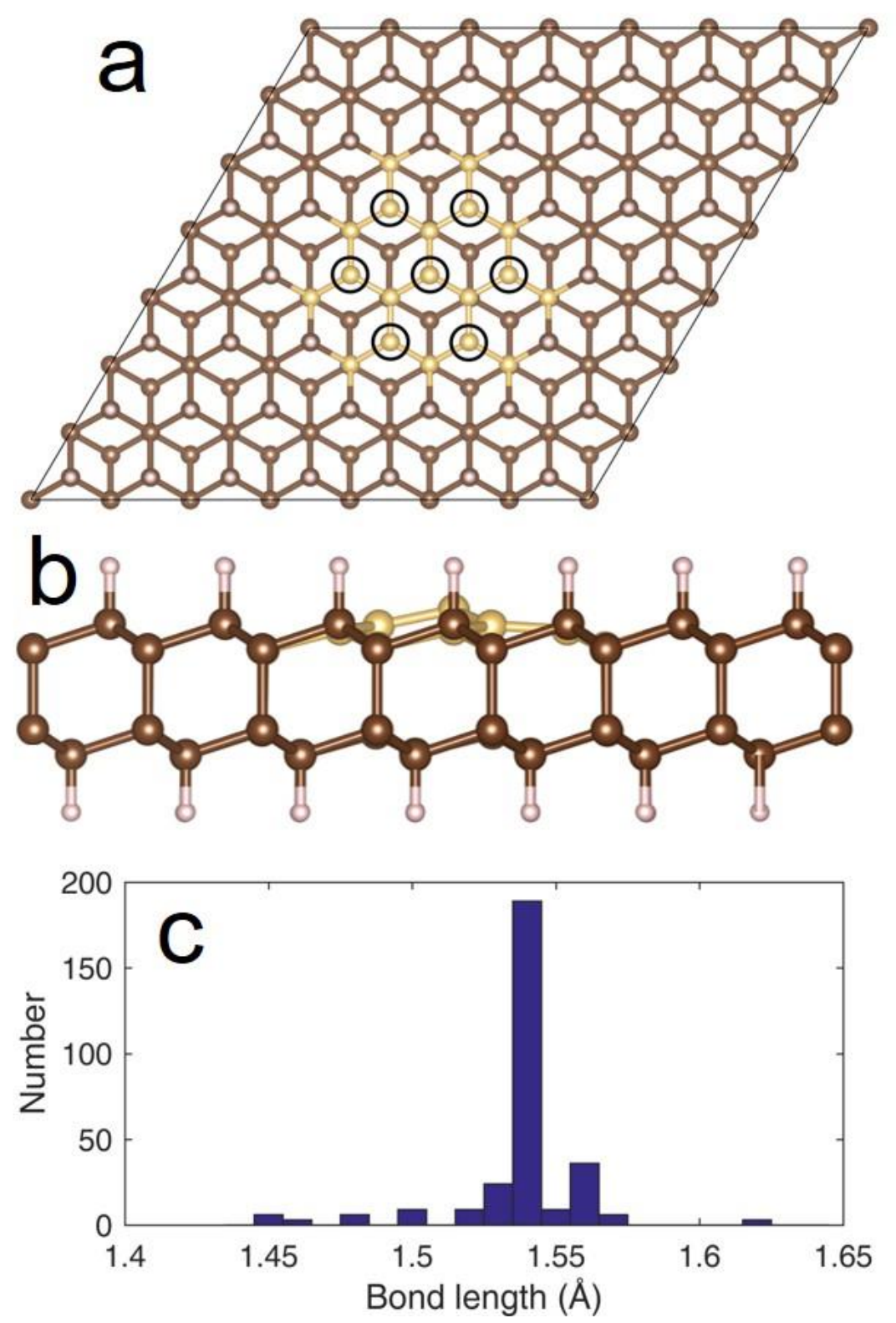

Figure 3: (a) Top view of the reconstructed unsaturated structure, with 7 missing hydrogen atoms on the top layer (position of the missing $H$ atoms is given by the black circles, and (b) side view. Carbon atoms involved in the reconstruction are given in yellow, while the others are brown and $H$ atoms are white. (c) Resulting $C$-C bond-length distribution. 